\title{
Ceftazidime/avibactam: a novel cephalosporin/ nonbeta-lactam beta-lactamase inhibitor for the treatment of complicated urinary tract infections and complicated intra-abdominal infections
}

\author{
This article was published in the following Dove Press journal: \\ Drug Design, Development and Therapy \\ 26 July 2016 \\ Number of times this article has been viewed
}

\author{
Jose A Hidalgo 1,2 \\ Celeste MVinluan ${ }^{1-3}$ \\ Nishaal Antony ${ }^{3}$ \\ 'UTEP/UT Austin Cooperative \\ Pharmacy Program, College of \\ Health Sciences, University of Texas \\ at El Paso, El Paso, ${ }^{2}$ Department of \\ Pharmacy, College of Pharmacy, The \\ University of Texas at Austin, Austin, \\ ${ }^{3}$ Department of Internal Medicine, \\ Texas Tech University Health Sciences \\ Center, El Paso, TX, USA
}

\begin{abstract}
There has been greater interest in developing additional antimicrobial agents due to the increasing health care costs and resistance resulting from bacterial pathogens to currently available treatment options. Gram-negative organisms including Enterobacteriaceae and Pseudomonas aeruginosa are some of the most concerning threats due to their resistance mechanisms: extended-spectrum beta-lactamase production and Klebsiella pneumoniae carbapenemase enzymes. Ceftazidime is a third-generation broad-spectrum cephalosporin with activity against $P$. aeruginosa and avibactam is a novel nonbeta-lactam beta-lactamase inhibitor. Avycaz ${ }^{\mathbb{R}}$, the trade name for this new combination antibiotic, restores the activity of ceftazidime against some of the previously resistant pathogens. Avycaz was approved in 2015 for the treatment of complicated urinary tract infections, including pyelonephritis, and complicated intra-abdominal infections with the addition of metronidazole in patients with little to no other treatment options. This review article assesses the clinical trials and data that led to the approval of this antibiotic, in addition to its spectrum of activity and limitations.
\end{abstract}

Keywords: ceftazidime/avibactam, Avycaz, complicated urinary tract infections, complicated intra-abdominal infections

\section{Introduction}

Increasing resistance to currently available antimicrobial agents has prompted a renewed interest in developing additional agents. According to the World Health Organization's (WHO) Global Report on Surveillance, five out of six WHO regions reported $>50 \%$ resistance of Escherichia coli against third-generation cephalosporins and six out of six WHO regions reported equivocal resistance rates of Klebsiella pneumoniae against third-generation cephalosporins. ${ }^{1}$ Within the hospital setting, there has been increasing rates of resistance against some of the most common pathogens that cause complicated urinary tract infections (cUTIs) and complicated intra-abdominal infections (cIAIs), which have resulted in longer lengths of stay and increased hospital costs. ${ }^{2}$

According to the United States Centers for Disease Control, some of the most concerning threats to antibiotic resistance include carbapenem-resistant Enterobacteriaceae, extended-spectrum beta-lactamase-producing Enterobacteriaceae, and multidrug resistant Pseudomonas aeruginosa. ${ }^{3}$ These gram-negative organisms are responsible for several infections including cIAIs and cUTIs. The mechanism of resistance of Enterobacteriaceae species and $P$. aeruginosa is primarily caused by their production
Correspondence: Celeste M Vinluan West Coast University, School of Pharmacy, 590 North Vermont Ave, Los Angeles, CA 90004, USA

Tel +l 323454506 I

Email cVinluan@westcoastuniversity.edu (c) (1) (5) 2016 Hidalgo et al. This work is published and licensed by Dove Medical Press Limited. The full terms of this license are available at https://www.dovepress.com/terms.php hereby accept the Terms. Non-commercial uses of the work are permitted without any furcher permission from Dove Medical Press Limited, provided the work is properly attributed. For permission for commercial use of this work, please see paragraphs 4.2 and 5 of our Terms (https://www.dovepress.com/terms.php). 
of extended-spectrum beta-lactamases and $K$. pneumoniae carbapenemase (KPC) enzymes. The production of these enzymes renders the current beta-lactamase inhibitors ineffective against these resistant gram-negative pathogens. ${ }^{3,4}$ Before ceftazidime/avibactam, the primary drug of choice for KPC infection was polymyxin, which has been known to have a severe side effect profile. ${ }^{5}$

Ceftazidime/avibactam $\left(\right.$ Avycaz $\left.^{\circledR}\right)$ is a combination cephalosporin and beta-lactamase inhibitor that was approved in February 2015. ${ }^{6}$ The presently available beta-lactamase inhibitors are ineffective against Ambler Class A carbapenemases and Class $\mathrm{C}$ enzymes. ${ }^{7-9}$ Avibactam, formerly known as NXL104, is a nonbeta-lactam beta-lactamase inhibitor that has potent activity against most Ambler Class A enzymes, which include narrow and extended-spectrum beta-lactamases and some carbapenemases, Ambler Class $\mathrm{C}$ enzymes, which include extended spectrum cephalosporinases, and some Class D enzymes, which include carbapenemases. ${ }^{10}$ In this article, the clinical microbiology, pharmacology, and the most recent clinical data on ceftazidime/avibactam (two Phase I, two Phase II, and Phase III trials $)^{11-15}$ are discussed.

\section{Clinical microbiology}

Avibactam has activity against extended-spectrum beta-lactamases, including $K$. pneumoniae-producing carbapenemases, Class A enzymes, and extended-spectrum cephalosporinases that contain the $A m p C$ gene, which are Class $\mathrm{C}$ enzymes. ${ }^{16}$ Avibactam also has some activity against carbapenemases produced from the OXA gene, which are Class D enzymes. Ceftazidime has broad-spectrum activity against gram-negative bacteria including $P$. aeruginosa. Consequently, the addition of avibactam to ceftazidime increases the spectrum of activity to organisms that produce beta-lactamase enzymes. ${ }^{17}$ It is important to recognize that the addition of a beta-lactamase inhibitor does not increase activity against anaerobic organisms, in contrast to other combination beta-lactam/beta-lactamase inhibitors.

The antimicrobial activity of ceftazidime/avibactam was tested against gram-negative bacilli that were collected from 73 medical centers throughout the US. ${ }^{18}$ The results of the study confirmed that the combination of ceftazidime/avibactam had potent activity against KPC-producing Enterobacteriaceae and meropenem-nonsusceptible $P$. aeruginosa, both of which are resistant to currently available beta-lactamase inhibitors (clavulanic acid, tazobactam, and sulbactam). ${ }^{18}$

The in vitro antibacterial activity of ceftazidime/ avibactam against a panel of clinically isolated $P$. aeruginosa strains was evaluated to determine the clinical utility of this combination. The minimum inhibitory concentration (MIC) is a diagnostic laboratory test used to determine the susceptibility of pathogenic microorganisms to antibiotics. The MIC is determined by the minimum concentration of an antibiotic that inhibits visible growth overnight. ${ }^{19}$ When tested in comparison to other available beta-lactams, the addition of avibactam was effective in lowering the MIC for aztreonam, piperacillin, and imipenem. However, the addition of avibactam to ceftazidime resulted in the largest increase in activity from the $126 P$. aeruginosa strains collected between 2006 and 2007. Only 65\% of isolates were susceptible to ceftazidime alone, but $94 \%$ were susceptible to the combination of ceftazidime/avibactam. ${ }^{16}$

The activity of ceftazidime/avibactam was also tested against Enterobacteriaceae and $P$. aeruginosa isolates that expressed beta-lactamase genes. The addition of avibactam to ceftazidime resulted in a fourfold to 512-fold reduction in the MIC of the isolates with beta-lactamase genes, compared to avibactam alone. ${ }^{20}$ However, there were three isolates that demonstrated no change in MIC upon the addition of avibactam to ceftazidime. The first isolate was from an $E$. coli strain containing the $b l a_{\mathrm{TEM}}$ gene, in which the MIC remained unchanged when tested against ceftazidime and ceftazidime/ avibactam. The second was a $P$. aeruginosa isolate containing the bla $a_{\mathrm{VIM}}$ gene, which is a Class B metallobeta-lactamase to which avibactam had no effect. The third exception was a $P$. aeruginosa isolate with the $b l a_{\mathrm{OXA}-23}$ gene, a member of the Class D carbapenemase-producing class, hence its resistance was unlikely to be caused by beta-lactamase as this class is unable to hydrolyze extended-spectrum cephalosporins, such as ceftazidime. ${ }^{20}$ One study has determined that ceftazidime/ avibactam is effective against $P$. aeruginosa strains that contain a derepressed $A m p C$ gene, which is intrinsically resistant to ceftazidime. ${ }^{21}$ These strains were incubated in fixed concentrations of avibactam along with increasing ceftazidime concentrations. The frequency of resistance to ceftazidime/avibactam was low in the three strains that were challenged. Additionally, the authors suggested that the risk of developing spontaneous resistance to the ceftazidime/ avibactam combination was low as their findings revealed that the rate of resistance was lower when compared to meropenem or imipenem. ${ }^{21}$

A previous study has described the extensive microbiology activity of ceftazidime/avibactam, but until recently there have been no KPC-producing $K$. pneumoniae isolates that have been reported to be resistant to the drug. One case report has been published that documented the first case of 
resistance to ceftazidime/avibactam in a KPC-3-expressing $K$. pneumoniae isolate. ${ }^{11}$ The carbapenem-resistant $K$. pneumoniae isolate was resistant to aztreonam, tobramycin, carbapenems, ampicillin/sulbactam, extended-spectrum cephalosporins, piperacillin/tazobactam, and ciprofloxacin. The isolate was also resistant to doxycycline, minocycline, chloramphenicol, and tigecycline. The exact mechanism of resistance for this KPC K. pneumoniae isolate to ceftazidime/ avibactam is uncertain and is under investigation. Despite the introduction of these enzymes are uncommon, the previous example of resistance warrants increased monitoring and surveillance for these isolates. ${ }^{11}$

\section{Pharmacokinetics}

Ceftazidime/avibactam is available as an intravenous (IV) formulation. The components of ceftazidime/avibactam are formulated in a $4: 1$ ratio containing $2,000 \mathrm{mg}$ of ceftazidime and $500 \mathrm{mg}$ of avibactam. ${ }^{22}$ The pharmacokinetics of both ceftazidime and avibactam are similar, in which their individual properties remain unchanged when both are coadministered. Ceftazidime and avibactam follow twocompartment linear kinetics with their maximum plasma concentration $\left(C_{\max }\right)$ and area under the curve increasing linearly with additional and higher doses. Both components of ceftazidime/avibactam are minimally protein bound with $<10 \%$ plasma protein binding. ${ }^{11}$ Ceftazidime (80\%-90\% of the dose) and avibactam are not metabolized and are primarily renally excreted in their unchanged form; the renal clearance for ceftazidime and avibactam is $115 \mathrm{~mL} / \mathrm{min}$ and $158 \mathrm{~mL} / \mathrm{min}$, respectively. ${ }^{6}$ In patients with normal renal function, the half-life $\left(t_{1 / 2}\right)$ for each component is relatively similar: 2.76 hours and 2.71 hours for ceftazidime and avibactam, respectively. ${ }^{6}$

\section{Chemical structure}

The basic structure of cephalosporins comprises of a fourmember $\beta$-lactam ring and a six-member dihydrothiazine ring. Different side chain substitutions at the three and seven positions have yielded the manufacture of several antibiotics in the cephalosporin class. ${ }^{23}$ Ceftazidime contains a pyridinium group at position 3 that increases activity against $P$. aeruginosa and its water solubility. ${ }^{23,24}$ At position 7, the aminothiadiazole ring provides activity against gram-negative bacilli, while the carboxypropyloxyimino group enhances its activity toward $P$. aeruginosa and increases its stability against beta-lactamase; however, it also consequently reduces its activity against Enterobacteriaceae. ${ }^{25-27}$ Avibactam is categorized into a new class of nonbeta-lactam beta-lactamase inhibitors, diazabicyclooctanes (DBOs). ${ }^{17}$

\section{Indications and usage}

Current clinical indications for ceftazidime/avibactam include the treatment of patients 18 years or older with cIAIs and cUTIs. ${ }^{6}$ However, there are ongoing clinical studies that are investigating additional indications for the use of ceftazidime/ avibactam. Full results from the Phase III trials and future studies are likely to expand the use of ceftazidime/avibactam to include hospitalized adults with nosocomial and ventilatorassociated pneumonia, hospitalized pediatric patients aged 3 months to 18 years with cIAIs, and cystic fibrosis patients with resistant respiratory $P$. aeruginosa infections. ${ }^{12,28,29}$

\section{Clinical pharmacology}

Ceftazidime/avibactam consists of an antibacterial betalactam, ceftazidime, and a beta-lactamase inhibitor, avibactam. Ceftazidime is a beta-lactam that exhibits bactericidal activity by inhibiting cell wall synthesis by binding to penicillin-binding proteins, resulting in bacterial cell death. ${ }^{30,31}$ Avibactam binds covalently to beta-lactamases at the active site, but this simple mechanism does not involve a rearrangement that other inhibitors with a beta-lactam moiety undergo after the opening of the four-membered beta-lactam ring. ${ }^{17}$ Avibactam is from a group of beta-lactamase inhibitors called DBOs, which are more potent when compared to the prototypical beta-lactam ring structure. For example, a significant amount of tazobactam and clavulanic acid is required to inhibit one beta-lactamase molecule, compared to only one to five molecules of avibactam. ${ }^{17}$ The increased efficiency and activity of the DBO molecule are due to the ring opening and being stabilized by residues that are close to the active site, causing polar interactions. In contrast, the opening of the four-ring beta-lactam structure undergoes rearrangement upon the opening of the ring, resulting in destabilization. This mechanism of reaction allows for a more efficient inhibition and broadens the activity against bacteria that would normally degrade ceftazidime. ${ }^{17}$

\section{Clinical trials}

\section{Phase I studies}

Two Phase I randomized, double-blinded, placebo-controlled studies were done to evaluate the safety, tolerability, and pharmacokinetics of avibactam both alone and in combination with ceftazidime. ${ }^{22}$ Subjects were included if they were healthy males aged 18 years to 45 years of age, had a body mass index of $18-27 \mathrm{~kg} / \mathrm{m}^{2}$, negative serology for HIV, 
hepatitis $\mathrm{B}$, hepatitis $\mathrm{C}$, negative urine drug screen, and no history of hypersensitivity to any medications. Additionally, no other medications were allowed beginning 14 days before the first dose until the end of the study; the only exception was $1,000 \mathrm{mg}$ of acetaminophen per day for a headache. Subjects were excluded if there were any electrocardiogram abnormalities, a presence of clinically significant illness 3 months before the beginning of the study, or any condition that would obstruct the absorption, distribution, metabolism, or excretion of any medication. ${ }^{22}$ Subjects were also excluded if there were any abnormal vital signs such as a diastolic blood pressure $>90 \mathrm{mmHg}$ and/or systolic blood pressure $>150 \mathrm{mmHg}$, any history of drug abuse 1 year prior to the study, smoking more than five cigarettes per day in the prior 3 months, consuming any products that contain grapefruit 7 days before the first dose, and blood or blood product donation $>500 \mathrm{~mL} 3$ months before the start of the study.

The first of these studies (NXL104-1001) was conducted using avibactam as a single ascending dose. Subjects were allotted to one of seven groups that consisted of avibactam doses ranging from $50 \mathrm{mg}$ to 2,000 $\mathrm{mg}$. Each group was made up of ten subjects: two subjects received placebo and eight received avibactam. After a 7-day washout period, subjects from the $250 \mathrm{mg}$ and $500 \mathrm{mg}$ groups received either placebo or a second avibactam dose that contained $1,000 \mathrm{mg}$ or $2,000 \mathrm{mg}$ of ceftazidime. ${ }^{22}$ The second study (NXL104-1002) was performed in two parts: the first part of the study was a multiple ascending dose study, which was made up of ten subjects in each one of the four dosing groups - avibactam $500 \mathrm{mg}$, $750 \mathrm{mg}$, or $1,000 \mathrm{mg}$, or avibactam $500 \mathrm{mg} /$ ceftazidime
$2,000 \mathrm{mg}$. Two subjects in each dosing group would receive placebo and eight subjects would receive avibactam every 8 hours for 5 days. The group receiving avibactam $500 \mathrm{mg} /$ ceftazidime $2,000 \mathrm{mg}$ received a 30 -minute IV infusion every 8 hours for 10 days. The second part of this study included eight subjects from a separate cohort who received avibactam $500 \mathrm{mg}$ IV or orally. ${ }^{22}$ There were no serious adverse events reported in either study. ${ }^{22}$ There were eleven treatment-emergent adverse effects (TEAEs), all of which were mild-to-moderate intensity, and all resolved spontaneously except one which was a moderate hematoma located at the injection site. No significant abnormalities in vital signs, hematology, electrocardiogram, biochemistry, and urinalysis were reported. Avibactam, when administered alone or in combination with ceftazidime, was well tolerated in the study dosages which ranged from $50 \mathrm{mg}$ to $2,000 \mathrm{mg}$. The two Phase I trials illustrate that the pharmacokinetic and safety profile of avibactam are not affected when given in combination with ceftazidime.

\section{Phase II studies}

Ceftazidime/avibactam was studied in two Phase II, randomized, double-blinded clinical trials. ${ }^{13,14}$ One study was conducted in patients with cIAIs, and the other study was performed in patients with cUTIs. Each study was designed to test the safety and efficacy of ceftazidime/avibactam when compared with a carbapenem. The similarities and differences between the two Phase II trials upon which ceftazidime/ avibactam gained its Food and Drug Administration approval are illustrated in Table 1.

Table I Comparison of safety and efficacy data of Phase II cUTI and Phase II clAI trials

\begin{tabular}{|c|c|c|c|c|c|c|}
\hline Study & Intervention & $\begin{array}{l}\text { Intervention } \\
\text { response }\end{array}$ & $\begin{array}{l}\text { Primary } \\
\text { pathogen } \\
\text { isolated }\end{array}$ & $\begin{array}{l}\text { Median duration } \\
\text { of treatment }\end{array}$ & Most common AEs & Limitations \\
\hline $\begin{array}{l}\text { Phase II } \\
(\mathrm{cUTI})^{14}\end{array}$ & $\begin{array}{l}\text { Ceftazidime/avibactam } \\
500 \mathrm{mg} / \mathrm{I} 25 \mathrm{mg} \text { IV q8h } \\
\text { Imipenem/cilastatin } \\
500 \mathrm{mg} \text { IV q6h }\end{array}$ & $\begin{array}{l}\text { Favorable clinical } \\
\text { response: } 24 / 28 \\
(85.7 \%)\end{array}$ & $\begin{array}{l}\text { E. coli } 25 / 27 \\
\text { isolates }(92.6 \%) \\
\text { E. coli } 33 / 35 \\
\text { isolates }(94.3 \%)\end{array}$ & $\begin{array}{l}5 \text { days (ceftazidime/ } \\
\text { avibactam) } \\
6 \text { days (imipenem/ } \\
\text { cilastatin) }\end{array}$ & $\begin{array}{l}\text { Headache, abdominal } \\
\text { pain, constipation, and } \\
\text { anxiety } \\
\text { Dizziness, injection/ } \\
\text { infusion site reaction, } \\
\text { abdominal pain/ } \\
\text { distension, and anxiety }\end{array}$ & $\begin{array}{l}\text { Formal sample size } \\
\text { calculation not performed } \\
\text { and not powered to } \\
\text { demonstrate noninferiority } \\
\text { to comparator }\end{array}$ \\
\hline \multirow[t]{2}{*}{$\begin{array}{l}\text { Phase II } \\
(\mathrm{clAl})^{13}\end{array}$} & $\begin{array}{l}\text { Ceftazidime/avibactam } \\
2,000 \mathrm{mg} / 500 \mathrm{mg} \text { IV } \\
\text { q8h plus metronidazole } \\
500 \mathrm{mg} \text { IV q8h }\end{array}$ & $\begin{array}{l}\text { Favorable clinical } \\
\text { response: } 80 / 87 \\
(92 \%)\end{array}$ & $\begin{array}{l}\text { E. coli } 105 / 105 \\
\text { isolates (100\%) }\end{array}$ & $\begin{array}{l}6 \text { days (ceftazidime/ } \\
\text { avibactam plus } \\
\text { metronidazole) }\end{array}$ & $\begin{array}{l}\text { Vomiting, nausea, } \\
\text { abdominal pain, and } \\
\text { pyrexia }\end{array}$ & $\begin{array}{l}\text { Study not statistically } \\
\text { powered to demonstrate } \\
\text { noninferiority to } \\
\text { comparator }\end{array}$ \\
\hline & $\begin{array}{l}\text { Meropenem I,000 mg IV } \\
\text { q8h plus placebo infusion }\end{array}$ & & $\begin{array}{l}\text { E. coli } 105 / 105 \\
\text { isolates (100\%) }\end{array}$ & $\begin{array}{l}6.5 \text { days } \\
\text { (meropenem) }\end{array}$ & $\begin{array}{l}\text { Pyrexia, wound } \\
\text { secretion, nausea, and } \\
\text { vomiting }\end{array}$ & \\
\hline
\end{tabular}

Note: Data from Lucasti et al ${ }^{13}$ and Vazquez et al. ${ }^{14}$

Abbreviations: AEs, adverse effects; clAl, complicated intra-abdominal infection; cUTI, complicated urinary tract infection; E. coli, Escherichia coli; IV, intravenous; qxh, every $x$ hours. 
In one Phase II randomized, double-blinded, activecontrolled trial, the safety and efficacy of ceftazidime/ avibactam plus metronidazole compared to meropenem was evaluated in 203 hospitalized patients with cIAIs. ${ }^{29}$ Patients with a confirmed diagnosis of cIAI that required surgical intervention and antibiotics were enrolled and randomized in a 1:1 ratio. Patients received either a combination dose of ceftazidime $2,000 \mathrm{mg}$ plus avibactam $500 \mathrm{mg}$ administered IV over 30 minutes every 8 hours plus a separate infusion of metronidazole $500 \mathrm{mg}$ administered over 1 hour every 8 hours, or meropenem 1,000 mg every 8 hours plus an infusion of placebo over 1 hour (Table 1). The primary end point of the study was clinical response at the test of cure (TOC) visit, 5 days to 14 days after treatment. Patients were eligible to participate if they were aged 18 years to 90 years with a cIAI requiring surgical intervention and antibiotics. The diagnoses that were accepted included diverticular disease with perforation or abscess, secondary peritonitis, intra-abdominal abscess with intraperitoneal involvement, cholecystitis with gangrenous rupture, or acute gastric or duodenal perforation. Patients were excluded if they had small bowel obstruction or ischemic bowel without perforation, abdominal wall abscess, or other coexisting infections that would interfere with the analysis of the study. Patients were also excluded if they had previously received antibiotic therapy within 72 hours of study therapy, abnormal liver function defined as aspartate transaminase greater than three times the upper limit of normal, or an infection caused by a pathogen with resistance to the study medication. Further exclusion criteria included patients with infections of the female genitalia or perinephric infections, survival anticipated to be less than the study period, sepsis with shock not responsive to IV fluids, APACHE II score $>25$, or renal dysfunction defined as creatinine clearance $(\mathrm{CrCl})<50 \mathrm{~mL} / \mathrm{min}$. Continued exclusion criteria included hematological or metastatic malignancy requiring chemotherapy, chronic cirrhosis or hepatitis, body mass index $>45 \mathrm{~kg} / \mathrm{m}^{2}$, absolute neutrophil count $<1,500 \mathrm{cell} / \mathrm{mm}^{3}$ (unless associated with infection), or immunocompromised status defined as HIV with AIDS or $\mathrm{CD}^{+} \mathrm{T}$-lymphocyte count $<2,000$ cells $/ \mathrm{mm}^{3}$. The final exclusion criteria included patients with a hemoglobin level $<10 \mathrm{~g} / \mathrm{dL}$, platelet count $<100,000$ cells $/ \mathrm{mm}^{3}$, splenectomy or chronic corticosteroid therapy defined as $>20 \mathrm{mg}$ of prednisone daily, patients who required additional systemic antibiotic therapy, and patients with 5 days to 14 days of therapy that were deemed unlikely to respond to treatment.

A total of 87 patients in the ceftazidime/avibactam plus metronidazole group and 90 patients in the meropenem group were clinically evaluable (CE). There were 68 and 76 patients who were microbiologically evaluable (ME), with a median duration treatment time of 6 days and 6.5 days in the ceftazidime/avibactam plus metronidazole and meropenem groups, respectively..$^{13}$ In the ceftazidime/avibactam plus metronidazole and meropenem groups, a clinical response was seen in $92 \%$ and $94.4 \%$, respectively, in the CE subgroup and $91.2 \%$ and $93.4 \%$, respectively, in the ME subgroup at TOC. The authors of the study concluded that there was no relationship between APACHE II scores at baseline and treatment outcome. However, it should be noted that the number of patients with an APACHE II score $>10$ was limited to 12 and eleven patients in the ceftazidime/avibactam plus metronidazole and meropenem group, respectively. Furthermore, response rates appeared to be similar between the two groups, independent of whether the infection was monomicrobial or polymicrobial in nature. ${ }^{13}$

A small prospective, randomized, investigator-blinded, Phase II trial compared the efficacy, safety, and tolerability of ceftazidime/avibactam and imipenem/cilastatin in adults who were hospitalized with a severe cUTI resulting from a gramnegative pathogen. ${ }^{14}$ This trial recruited patients from 26 different centers located throughout five countries. Patients in the study were randomized to receive either ceftazidime $500 \mathrm{mg}$ plus avibactam $125 \mathrm{mg}$ infused IV over 30 minutes every 8 hours or cilastatin/imipenem $500 \mathrm{mg}$ infused IV over 30 minutes every 6 hours. The median duration of therapy was 5 days in the ceftazidime/avibactam group and 6 days in the cilastatin/imipenem group. The primary end point of the study was a positive microbiological response in the ME patients at the TOC visit, which was 5 days to 9 days after the last dose of therapy. The secondary end point of the study was a microbiological response in the ME patients at the end of IV therapy and late follow-up visit (LFU), 4 weeks to 6 weeks after therapy. Patients were included if they were between the ages of 18 years and 90 years and were diagnosed with acute pyelonephritis or cUTI caused by a gram-negative pathogen that was deemed to be a serious infection requiring parenteral therapy. Acute pyelonephritis was defined to include patients who were febrile (oral body temperature $>37.8^{\circ} \mathrm{C}$ ) with the presence of chills beginning 12 hours into the study, pyuria, flank pain, and positive urine culture with $\geq 10^{5} \mathrm{CFU} / \mathrm{mL}$ caused by a pathogen with a known susceptibility to the study drugs. Patients with cUTIs were defined by having signs and symptoms that were consistent with a UTI. This included an oral body temperature $>37.8^{\circ} \mathrm{C}$, suprapubic pain, nausea/vomiting, dysuria, frequency, urgency, incontinence, costovertebral angle tenderness, and a positive urine culture 
with $\geq 10^{5} \mathrm{CFU} / \mathrm{mL}$ caused by a pathogen with known or considered susceptibility to the study drugs. Patients were excluded if they had previously received at least one dose of another systemic antibiotic 48 hours before the admission urine culture, if the cUTI was caused by a pathogen with known resistance to any of the study drugs, full obstruction of the urinary tract, fungal UTI, presence of vesicoureteral reflux or ileal loops, pregnant or breast-feeding women, patients with a known history of hypersensitivity to the study medications, patients with permanent indwelling catheter, and patients believed unlikely to survive the study trial period.

The CE population had a favorable clinical response in all patients at the end of IV therapy. A favorable clinical response among the $\mathrm{CE}$ population at the TOC visit was observed in $24 / 28(85.7 \%)$ of the ceftazidime/avibactam group and 29/36 (80.6\%) in the imipenem/cilastatin arm. Results for these positive clinical outcomes are presented in Table 1. In the ME population, there was a $70.4 \%$ and $71.4 \%$ positive clinical response at TOC visit, $96.2 \%$ and $100 \%$ positive clinical response at the end of IV therapy, and a $57.7 \%$ and $60 \%$ positive response at the LFU visit in the ceftazidime/avibactam and imipenem/cilastatin groups, respectively. Comprehensively in the ME population, there was a positive microbiological and clinical response in $66.7 \%$ and $60 \%$ of patients at the TOC visit and $53.8 \%$ and $60 \%$ at the LFU visit in the ceftazidime/avibactam and imipenem/ cilastatin groups, respectively. The authors of the study noted that despite the small sample size, six of seven patients $(85.7 \%)$ and nine of eleven patients $(81.8 \%)$ in the ME group who had a ceftazidime-resistant pathogen at the TOC visit showed a positive microbiological response with ceftazidime/ avibactam and imipenem/cilastatin, respectively. ${ }^{14}$

\section{Phase III studies}

Results from two new Phase III trials, RECLAIM-1 and RECLAIM-2, were only available in abstract form. The two studies were analyzed as one dataset and were conducted in patients with cIAIs that compared ceftazidime/avibactam plus metronidazole to meropenem. ${ }^{15,32}$ Both studies used the randomization of ceftazidime 2,000 $\mathrm{mg}$ plus avibactam $500 \mathrm{mg}$ every 8 hours and metronidazole $500 \mathrm{mg}$ every 8 hours or meropenem 1,000 mg every 8 hours. However, the ceftazidime/avibactam infusion was changed to 2 hours compared to the 30 minutes that was conducted in the Phase II trial. ${ }^{14}$ The primary efficacy end point of the RECLAIM study was clinical response at the TOC visit, which was assessed at 28 days to 35 days after randomization. The study randomized a total of 1,066 patients, and the subjects were followed after a period of 2 years. The overall cure rate was achieved in $82 \%$ patients receiving ceftazidime/avibactam and $85 \%$ for meropenem ( $-3.5 ; 95 \% \mathrm{CI}$ : -8.6 to 1.6$)$, showing noninferiority between the two treatments. ${ }^{15,32}$ Results from this Phase III cIAI trial showed meropenem had decreased mortality compared with ceftazidime/avibactam plus metronidazole ( $1.5 \%$ versus $2.5 \%$, respectively). ${ }^{6}$ Clinical cure rates were reduced in patients with moderate renal impairment ( $\mathrm{CrCl} 30-50 \mathrm{~mL} / \mathrm{min}$ ) who received ceftazidime/avibactam compared to those patients who received meropenem, $45 \%$ and $74 \%$, respectively. Within this group of patients with a $\mathrm{CrCl} 30-50 \mathrm{~mL} / \mathrm{min}$, death occurred in $25.8 \%$ of participants who received ceftazidime/avibactam plus metronidazole compared with $8.6 \%$ of participants who received meropenem, which may be attributed to participants receiving a 33\% lower daily dose than is recommended. ${ }^{6,32}$ This difference in clinical cure rates was not seen in patients with a normal renal function or mild renal impairment ( $\mathrm{CrCl}>50 \mathrm{~mL} / \mathrm{min}$ ), suggesting insufficient dosing may have caused the lower cure rates. ${ }^{15}$

\section{Adverse drug reactions}

Based on Phase I and Phase II trials, ceftazidime/avibactam is generally well tolerated. All subjects completed the study with no patients requiring discontinuation from the study due to adverse effects (AEs). There were six TEAEs reported in four subjects in the single ascending dose arm of the study. Only one AE was considered moderate in intensity and it resolved spontaneously while the others were considered only mild in intensity. The TEAEs that were considered related to the therapy included: postural dizziness, anxiety, abdominal pain, somnolence, and sense of oppression. In the multiple ascending dose arm, the only TEAE observed was a moderate hematoma at the injection site. ${ }^{22}$ The AEs reported from the two Phase II trials also indicated that ceftazidime/ avibactam was well tolerated when compared with meropenem and imipenem/cilastatin for cIAI and cUTI. ${ }^{13,14}$ Observations of AEs were reported in $64.4 \%$ of patients who received ceftazidime/avibactam plus metronidazole, compared to $57.8 \%$ of patients who received meropenem. The most common TEAEs reported were an increased frequency of vomiting (13.9\%), nausea $(9.9 \%)$, pyrexia $(8.9 \%)$, and abdominal pain $(7.9 \%)$ in the ceftazidime/avibactam plus metronidazole group. However, most of the AEs were mild to moderate in intensity. In a separate Phase II study, $67.6 \%$ of patients in the ceftazidime/avibactam group and $76.1 \%$ of patients in the imipenem/cilastatin group reported AEs. Based on 68 subjects in the group receiving 
ceftazidime/avibactam, the most common TEAE included a headache (19.1\%), abdominal pain (14.7\%), constipation $(10.3 \%)$, anxiety $(10.3 \%)$, and diarrhea $(8.8 \%){ }^{14}$

\section{Drug interactions}

Ceftazidime/avibactam retains its clinical efficacy when exposed to levels of pulmonary surfactant that would otherwise inhibit the bactericidal effects of daptomycin. As well, there have been no known antagonistic effects of ceftazidime/ avibactam when used in conjunction with other antibiotics for the treatment of nosocomial-associated pneumonias. ${ }^{5,33}$ However, avibactam, in vitro, is a substrate of OAT1 and OAT3 transporters. The coadministration of probenecid with avibactam resulted in the inhibition of $\sim 56 \%$ to $70 \%$ of the uptake of avibactam. Therefore, the coadministration of ceftazidime/ avibactam and probenecid is not recommended. ${ }^{6,34}$ Furthermore, administration with oral contraceptive pills is not recommended due to decreased intestinal uptake of estrogen causing subtherapeutic levels of estrogen.,35

\section{Contraindications}

Contraindications for the use of ceftazidime/avibactam include known severe hypersensitivity reactions to Avycaz, ceftazidime, avibactam, or other members of the cephalosporin class. ${ }^{6}$

\section{Warnings and precautions}

Hypersensitivity and skin reactions, Clostridium difficileassociated diarrhea, and central nervous system adverse reactions that were more prevalent in renally impaired patients, are concerns with ceftazidime/avibactam. Possible development of drug-resistant bacteria should also be recognized with use of ceftazidime/avibactam. ${ }^{6,35}$ In the Phase III cIAI trial, patients with a baseline $\mathrm{CrCl}$ of $30 \mathrm{~mL} / \mathrm{min}$ to $50 \mathrm{~mL} / \mathrm{min}$ demonstrated a lower clinical response to ceftazidime/avibactam. $\mathrm{CrCl}$ should be monitored, and the dose should be adjusted accordingly in patients with changing renal function. ${ }^{6,15}$

\section{Special populations}

This formulation has not been studied and is not currently approved for patients younger than 18 years of age. The dose and/or interval of ceftazidime/avibactam should be adjusted in patients with renal impairment $(\mathrm{CrCl}<50 \mathrm{~mL} / \mathrm{min}$ based on the Cockcroft and Gault formula). Cephalosporins as a class are considered pregnancy risk category $\mathrm{B}$ and ceftazidime is excreted in breast milk. It is unknown if avibactam is excreted in breast milk. ${ }^{6}$

\section{Conclusion}

Clinicians have fewer antibiotic options to treat patients as drug-resistant gram-negative pathogens continue to rise. The introduction of avibactam, a nonbeta-lactam beta-lactamase inhibitor, acts differently than other beta-lactamase inhibitors and broadens the activity against bacteria that would normally degrade ceftazidime such as Amp C beta-lactamases, carbapenemase-producing Entera bacteria gene, and multidrug resistant Pseudomonas aeruginosa. ${ }^{5,10}$ Ceftazidime/ avibactam is currently only indicated for use in patients with cIAIs (in combination with metronidazole) and cUTIs, including pyelonephritis, and should be reserved for patients who have no alternate treatment options because of antibiotic resistance. Judicious use of antibiotic stewardship should be used when using this medication to prevent the incidence of drug resistance. Ceftazidime/avibactam has been shown to be well tolerated in several clinical trials, with only mild-to-moderate intensity AEs reported. Future indications and resistance mechanisms remain to be seen. However, the broad spectrum of activity that ceftazidime/ avibactam provides is an important advancement in the treatment of patients with cUTIs and cIAIs with limited treatment options.

\section{Acknowledgment}

Celeste M Vinluan is currently affiliated with West Coast University at the time of publication.

\section{Disclosure}

The authors report no conflicts of interest in this work.

\section{References}

1. World Health Organization [webpage on the Internet]. Antimicrobial Resistance: Global Report on Surveillance, 2014; 2014. Available from: http://apps.who.int/iris/bitstream/10665/112642/1/9789241564748_eng. pdf?ua=1. Accessed March 20, 2016.

2. Cosgrove SE. The relationship between antimicrobial resistance and patient outcomes: mortality, length of hospital stay, and health care costs. Clin Infect Dis. 2006;42(suppl 2):S82-S89.

3. Centers for Disease Control and Prevention [webpage on the Internet]. Antibiotic Resistance Threats in the United States, 2013; 2013. Available from: http://www.cdc.gov/drugresistance/threat-report-2013/pdf/ ar-threats-2013-508.pdf. Accessed February 3, 2016.

4. Chen J, Shang X, Hu F, et al. Beta-lactamase inhibitors: an update. Mini Rev Med Chem. 2013;13(13):1846-1861.

5. Sharma R, Park TE, Moy S. Ceftazidime-avibactam: a novel cephalosporin/ $\beta$-lactamase inhibitor combination for the treatment of resistant gram-negative organisms. Clin Ther. 2016;38(3):431-444.

6. Avycaz (Ceftazidime-Avibactam) [package insert]. Cincinnati, OH: Forest Pharmaceuticals; 2015.

7. Castanheira M, Farrell SE, Wanger A, Rolston KV, Jones RN, Mendes RE. Rapid expansion of KPC-2-producing Klebsiella pneumoniae isolates in two Texas hospitals due to clonal spread of ST258 and ST307 lineages. Microb Drug Resist. 2013;19(4):295-297. 
8. Tzouvelekis LS, Markogiannakis A, Psichogiou M, Tassios PT, Daikos GL. Carbapenemases in Klebsiella pneumoniae and other enterobacteriaceae: an evolving crisis of global dimensions. Clin Microbiol Rev. 2012;25(4): $682-707$.

9. Woodford N, Turton JF, Livermore DM. Multiresistant gram-negative bacteria: the role of high-risk clones in the dissemination of antibiotic resistance. FEMS Microbiol Rev. 2011;35(5):736-755.

10. Aktaş Z, Kayacan C, Oncul O. In vitro activity of avibactam (NXL104) in combination with $\beta$-lactams against gram-negative bacteria, including OXA-48 $\beta$-lactamase-producing Klebsiella pneumoniae. Int J Antimicrob Agents. 2012;39(1):86-89.

11. Humphries RM, Yang S, Hemarajata P, et al. First report of ceftazidimeavibactam resistance in a KPC-3-expressing Klebsiella pneumoniae isolate. Antimicrob Agents Chemother. 2015;59(10):6605-6607.

12. AstraZeneca; PRA Health Sciences [webpage on the Internet]. Evaluation of Safety, Pharmacokinetics and Efficacy of CAZ-AVI with Metronidazole in Children Aged 3 Months to 18 Years Old with Complicated Intra-abdominal Infections (cIAIs). In: ClinicalTrials. gov [Internet]. Bethesda, MD: National Library of Medicine (US); 2000 [cited March 24, 2016]. Available from: http://clinicaltrials.gov/ show/NCT02475733.

13. Lucasti C, Popescu I, Ramesh M, Lipka J, Sable C. Comparative study of the efficacy and safety of ceftazidime/avibactam plus metronidazole versus meropenem in the treatment of complicated intra-abdominal infections in hospitalized adults: results of a randomized, double-blind, Phase II trial. J Antimicrob Chemother. 2013;68(5):1183-1192.

14. Vazquez J, González Patzán L, Stricklin D, et al. Efficacy and safety of ceftazidime-avibactam versus imipenem-cilastatin in the treatment of complicated urinary tract infections, including acute pyelonephritis, in hospitalized adults: results of a prospective, investigator-blinded, randomized study. Curr Med Res Opin. 2012;28(12):1921-1931.

15. Mazuski JE, Gasnik L, Amstrong J, et al. Efficacy and safety of ceftazidime-avibactam plus metronidazole versus meropenem in the treatment of complicated intra-abdominal infection-results from a Phase III program (Abstract O191). 25th European Congress of Clinical Microbiology and Infectious Disease (ECCMID); Copenhagen: 2015.

16. Levasseur P, Girard AM, Claudon M, et al. In vitro antibacterial activity of the ceftazidime-avibactam (NXL104) combination against Pseudomonas aeruginosa clinical isolates. Antimicrob Agents Chemother. 2012;56(3):1606-1608.

17. Coleman K. Diazabicyclooctanes (DBOs): a potent new class of non$\beta$-lactam $\beta$-lactamase inhibitors. Curr Opin Microbiol. 2011;14(5): $550-555$.

18. Sader H, Castanheira M, Flamm R, Farrell DJ, Jones RN. Antimicrobial activity of ceftazidime-avibactam against gram-negative organisms collected from U.S. medical centers in 2012. Antimicrob Agents Chemother. 2013;58(3):1684-1692.

19. Andrews JM. Determination of minimum inhibitory concentrations. J Antimicrob Chemother. 2001;48(suppl 1):5-16.

20. Keepers TR, Gomez M, Celeri C, Nichols WW, Krause KM. Bactericidal activity, absence of serum effect, and time-kill kinetics of ceftazidimeavibactam against $\beta$-lactamase-producing enterobacteriaceae and Pseudomonas aeruginosa. Antimicrob Agents Chemother. 2014;58(9): $5297-5305$
21. Lahiri SD, Walkup GK, Whiteaker JD, et al. Selection and molecular characterization of ceftazidime/avibactam-resistant mutants in Pseudomonas aeruginosa strains containing derepressed AmpC. J Antimicrob Chemother. 2015;70(6):1650-1658.

22. Merdjan H, Rangaraju M, Tarral A. Safety and pharmacokinetics of single and multiple ascending doses of avibactam alone and in combination with ceftazidime in healthy male volunteers: results of two randomized, placebo-controlled studies. Clin Drug Investig. 2015;35(5):307-317.

23. Beale J. Antibacterial antibiotics. In: Beale J, Block J, editors. Wilson and Gisvold's Textbook of Organic Medicinal and Pharmaceutical Chemistry. 12th ed. Baltimore: Lippincott Williams \& Wilkins; 2011:258-329.

24. Caprile K. The cephalosporin antimicrobial agents: a comprehensive review. J Vet Pharmacol Ther. 1988;11(1):1-32.

25. Dunn G. Ceftizoxime and other third-generation cephalosporins: structureactivity relationships. J Antimicrob Chemother. 1982;10(suppl C): $1-10$.

26. Neu H. $\beta$-Lactamase stability of cefoxitin in comparison with other $\beta$-lactam compounds. Diagn Microbiol Infect Dis. 1983;1(4):313-316.

27. Neu H. $\beta$-lactam antibiotics: structural relationships affecting in vitro activity and pharmacologic properties. Clin Infect Dis. 1986;8(suppl 3): S237-S259.

28. AstraZeneca [webpage on the Internet]. A Study Comparing CeftazidimeAvibactam versus Meropenem in Hospitalized Adults with Nosocomial Pneumonia. In: ClinicalTrials.gov [Internet]. Bethesda, MD: National Library of Medicine (US); 2000 [cited March 24, 2016]. Available from: http://clinicaltrials.gov/show/NCT01808092. Accessed June 10, 2016.

29. University of Southern California [webpage on the Internet]. SteadyState Pharmacokinetics of Ceftazidime/Avibactam in Cystic Fibrosis. In: ClinicalTrials.gov [Internet]. Bethesda, MD: National Library of Medicine (US); 2000 [cited March 24, 2016]. Available from: http:// clinicaltrials.gov/show/NCT02504827. Accessed June 10, 2016.

30. Vollmer W, Blanot D, De Pedro M. Peptidoglycan structure and architecture. FEMS Microbiol Rev. 2008;32(2):149-167.

31. Sauvage E, Kerff F, Terrak M, Ayala JA, Charlier P. The penicillinbinding proteins: structure and role in peptidoglycan biosynthesis. FEMS Microbiol Rev. 2008;32(2):234-258.

32. Mawal Y, Critchley IA, Riccobene TA, Talley AK. Ceftazidimeavibactam for the treatment of complicated urinary tract infections and complicated intra-abdominal infections. Expert Rev Clin Pharmacol. 2015;8(6):691-707.

33. Dallow J, Otterson LG, Huband MD, Krause KM, Nichols WW. Microbiological interaction studies between ceftazidime-avibactam and pulmonary surfactant and between ceftazidime-avibactam and antibacterial agents of other classes. Int J Antimicrob Agents. 2014;44(6): $552-556$.

34. Vishwanathan K, Mair S, Gupta A, et al. Assessment of the mass balance recovery and metabolite profile of avibactam in humans and in vitro drugdrug interaction potential. Drug Metab Dispos. 2014;42(5):932-942.

35. Fortaz [package insert]. Schaumburg, Illinois: Sagent Pharmaceuticals Inc.; 2014.
Drug Design, Development and Therapy

\section{Publish your work in this journal}

Drug Design, Development and Therapy is an international, peerreviewed open-access journal that spans the spectrum of drug design and development through to clinical applications. Clinical outcomes, patient safety, and programs for the development and effective, safe, and sustained use of medicines are a feature of the journal, which

\section{Dovepress}

has also been accepted for indexing on PubMed Central. The manuscript management system is completely online and includes a very quick and fair peer-review system, which is all easy to use. Visit http://www.dovepress.com/testimonials.php to read real quotes from published authors. 